\title{
Search for Alzheimer's disease biomarkers in blood cells: hypotheses-driven approach
}

\author{
Joanna Wojsiat ${ }^{1}$, Katarzyna Laskowska-Kaszub¹, Anna Mietelska-Porowska1 \& Urszula \\ Wojda*,1 \\ ${ }^{1}$ Laboratory of Preclinical Testing of Higher Standard, Nencki Institute of Experimental Biology, Polish Academy of Science, Pasteur \\ 3 St., 02-093 Warsaw, Poland \\ * Author for correspondence: Tel.: 48225892 578; Fax: 048225892 141; u.wojda@nencki.gov.pl
}

Current Alzheimer's disease (AD) diagnostics is based on cognitive testing, and detecting amyloid $A \beta$ and $\tau$ pathology by brain imaging and assays of cerebrospinal fluid. However, biomarkers identifying complex pathways contributing to pathology are lacking, especially for early AD. Preferably, such biomarkers should be more cost-effective and present in easily available diagnostic tissues, such as blood. Here, we summarize the recent findings of potential early AD molecular diagnostic biomarkers in blood platelets, lymphocytes and erythrocytes. We review molecular alterations which refer to such main hypotheses of $A D$ pathogenesis as amyloid cascade, oxidative and mitochondrial stress, inflammation and alterations in cell cycle regulatory molecules. The major advantage of such biomarkers is the potential ability to indicate individualized therapies in AD patients.

First draft submitted: 7 February 2017; Accepted for publication: 30 June 2017; Published online:

4 October 2017

Keywords: Alzheimer's disease $\bullet$ amyloid $\bullet$ cell-based biomarkers $\bullet$ early diagnostics $\bullet$ immune functions $\bullet$ lymphocytes $\bullet$ mitochondrial stress $\bullet$ oxidative stress $\bullet$ platelets $\bullet$ red blood cells

Alzheimer's disease $(\mathrm{AD})$ is the most prevalent age-related dementia worldwide and one of the major unmet, increasing medical and economic problems of the modern world. As of 2015, there were an estimated 46.8 million people with dementia worldwide. This number will increase to an estimated 74.7 million in 2030 [1].

Among the neuropathological hallmarks of $\mathrm{AD}$ are: the progressive loss of brain neurons, synaptic degeneration and the deposition of extracellular amyloid plaques and intracellular neurofibrillary tangles (NFTs) in such regions of the brain as the hippocampus, cortex and amygdala. Amyloid plaques consist mainly of 40- and 42-amino-acid $A \beta$ peptides (A $\beta 40$ and $A \beta 42$ ). Peptides are proteolytic cleavage products of the $A \beta$ protein precursor (APP) but with no fully elucidated biological function. NFTs are deposits of paired helical filaments composed mainly of hyperphosphorylated $\tau$ protein, a microtubule-stabilizing protein that maintains neuronal cell structure and axonal transport. Nevertheless, neither senile plaques nor NFTs are absolute hallmarks of AD dementia, because cognitively intact aged individuals may exhibit both pathological changes upon postmortem brain examination or as assessed by positron emission tomography amyloid and $\tau$ imaging agents (amyloid-PET and $\tau$-PET) [2-5].

Clinical AD manifests by aggravating dysfunction and weakening functional cognitive processes, linked to brain neuron loss that is both progressive and permanent [4], and that within several years leads to total dependence on the caregiver and eventually to death. Advances in our knowledge of AD have shown that clinical symptoms usually develop after a long preclinical pathogenic process, lasting for decades, making early $\mathrm{AD}$ detection in asymptomatic patients a subject of great interest $[4,5]$. Increasing proof demonstrates that the therapeutic methods' effectiveness is strictly contingent on the early diagnosis of $\mathrm{AD}$, before the occurrence of massive neuron loss and dementia. This highlights the key importance of biochemical biomarkers for early AD diagnostics.

\section{AD diagnostics \& demand for novel, improved biomarkers}

The broadly accepted recommendations for AD diagnosis come from the US National Institute of Aging and AD Association, the National Institute of Neurological and Communicative Disorders and Stroke and the AD and Related Disorders Association [6-8]. They differentiate subsequent phases of AD: a preclinical stage that is 
Table 1. Main advantages and disadvantages of blood based compared with cerebrospinal fluid-based and brain imaging Alzheimer's disease biomarkers.

\begin{tabular}{|c|c|c|}
\hline Biomarker source/assay type & Advantages & Disadvantages \\
\hline Blood-based biomarkers & $\begin{array}{l}\text { - Easy available for analysis } \\
\text { - Noninvasive procedures involved } \\
\text { - Cost-effective assays involved } \\
\text { - Multiple assessments possible } \\
\text { - Broad applications in diagnostics and monitoring } \\
\text { therapeutic outcomes }\end{array}$ & $\begin{array}{l}\text { - Interindividual variability } \\
\text { - Variability of analytical procedures across diagnostic } \\
\text { laboratories } \\
\text { - Possible analytical errors } \\
\text { - Normal biomarker range difficult to establish }\end{array}$ \\
\hline CSF-based biomarkers & $\begin{array}{l}\text { - Reflect disease progression and pathological changes } \\
\text { in the brain } \\
\text { - Do not require special laboratory equipment or } \\
\text { sophisticated procedures }\end{array}$ & $\begin{array}{l}\text { - Invasive lumbar puncture involved } \\
\text { - Assessment not always feasible, especially in elderly } \\
\text { patients } \\
\text { - Variability of analytical procedures across diagnostic } \\
\text { laboratories } \\
\text { - Possible analytical errors } \\
\text { - Normal biomarker range difficult to establish }\end{array}$ \\
\hline $\begin{array}{l}\text { Brain imaging biomarkers (amyloid-PET, } \\
\tau-\mathrm{PET} \text { ) }\end{array}$ & $\begin{array}{l}\text { - High early diagnosis capacity } \\
\text { - Directly reveals changes in the brain }\end{array}$ & $\begin{array}{l}\text { - High assay costs } \\
\text { - Requirement for sophisticated equipment and } \\
\text { know-how } \\
\text { - Techniques not available in all clinical settings }\end{array}$ \\
\hline
\end{tabular}

asymptomatic, a symptomatic predementia and AD dementia. Mild cognitive impairment (MCI) that is due to $\mathrm{AD}$ is also specified. In a clinic, an $\mathrm{AD}$ diagnosis is based mainly on cognitive testing and can be supported by brain imaging, in particular using amyloid-PET and $\tau$-PET, by cerebrospinal fluid (CSF) biomarkers: A $\beta$ peptides and total and hyperphosphorylated $\tau$ protein, and by genetic tests for APP and presenilins.

The advent of CSF and imaging biomarkers allowed for testing them for preclinical AD. The turning point for the acceptance of these biomarkers as indicators of preclinical $\mathrm{AD}$ were the results of the longitudinal study in carriers of rare familial $\mathrm{AD}(\mathrm{FAD})$ autosomal-dominant mutations in protein-encoding genes participating in the production of $A \beta$, over two decades before the onset of dementia symptoms [9]. Presently preclinical AD can be diagnosed based on the presence of both $\mathrm{A} \beta$ and $\tau$ biomarkers in the CSF or brain [5]. However, FAD is not fully representative for sporadic $\mathrm{AD}$, the latter being more complex and heterogeneous. This is reflected in cohorts without FAD mutations, where progression from preclinical AD defined based on positive $\tau$ and $A \beta$ markers to full $\mathrm{AD}$ dementia proved to be uncertain and dependent on the presence of other factors contributing to AD pathology. The risk of progressing to $\mathrm{AD}$ in such individuals, or individuals with only one biomarker positive, $\mathrm{A} \beta$ or $\tau$, is currently difficult to assess and biomarkers of such risk are missing [5]. These recent findings underlie the need for a broader panel of biomarkers which will allow not only to detect major hallmarks of AD such as $\mathrm{A} \beta$ and $\tau$, but also to detect $\mathrm{AD}$ risk factors for those in preclinical $\mathrm{AD}$, and which will report the type of contributing complex pathomechanisms in patients with clinical symptoms.

A biomarker is understood as an objectively measurable molecular indicator of a disease process, characteristic for a given stage of the disease, or/and indicator of a disease modification by therapeutic intervention. A good biomarker should fulfill the criteria of specificity and sensitivity, and should allow for the differentiation of AD from overlapping conditions, such as aging-related changes or dementia due to other neurodegenerative diseases. As indicated by the US FDA, for clinical applications, the biological samples in which the biomarker is to be measured should be easy to obtain in a safe procedure, preferably noninvasive, and the laboratory methods must be reliable, stable and cost-effective. In this light, while the recent development of brain imaging technologies and PET AD tracers as well as assays addressing the 'AD signature' in CSF reflect significant progress in AD diagnosis [4,5], these biomarkers do not fulfill all optimal diagnostic criteria for clinical practice (Table 1). Overall, it becomes clear that progress in $\mathrm{AD}$ diagnostics relies on identifying novel diagnostic biomarkers for $\mathrm{AD}$, especially for the early disease phase, that are more cost-effective and in more easily available diagnostic tissues, such as blood (Table 1).

As blood-based biomarkers minimize the risk and discomfort for patients and therefore broaden its applicability in an aging population, a blood-based assay can become the primary tool and the first step in a multistage AD diagnostic process, to identify those who should undergo further testing, involving more costly procedures and sophisticated equipment. Moreover, blood-based tests can be useful for large-scale diagnostics in clinics lacking specialized equipment, especially now when the threat of AD becomes global. A blood AD test could also be useful for monitoring therapeutic outcomes, especially for repeated measures. 
Table 2. Biomarker potential of circulating molecules in the cerebrospinal fluid and blood.

\begin{tabular}{|c|c|c|}
\hline Marker & CSF & Blood plasma/serum \\
\hline Tau-total $^{\dagger}$ & Significantly elevated in AD & $\begin{array}{l}\text { Significantly changed/considerable variability } \\
\text { between studies }\end{array}$ \\
\hline$A \beta 42^{\dagger}$ & Significantly decreased in AD & Not significantly different \\
\hline Tau-phospho ${ }^{\dagger}$ & Significantly elevated in AD & - \\
\hline YKL-40 & Significantly elevated in $A D$ & $\begin{array}{l}\text { Not significantly different/possible trend for } \\
\text { elevation in } A D\end{array}$ \\
\hline VLP-1 & Significantly elevated in $A D$ & - \\
\hline NFL & Significantly elevated in $A D$ & - \\
\hline Neurogranin & Significantly elevated in AD & Not significantly different \\
\hline hFABP & Significantly elevated in AD & - \\
\hline NSE & $\begin{array}{l}\text { Significantly changed/considerable variability between } \\
\text { studies }\end{array}$ & - \\
\hline MCP-1 & Marginal effect/slightly higher in $A D$ & Not significantly different \\
\hline STREM2 & Marginal effect/slightly higher in AD & - \\
\hline Albumin ratio & Marginal effect/slightly higher in AD & Marginal effect/slightly higher in AD \\
\hline$A \beta 40$ & Not significantly different & Not significantly different \\
\hline$A \beta 38$ & Not significantly different & - \\
\hline GFAP & Not significantly different & - \\
\hline SAPP $\beta$ & Not significantly different & - \\
\hline $\operatorname{sAPP} \alpha$ & Not significantly different & - \\
\hline \multicolumn{3}{|c|}{$\begin{array}{l}\text { †Biomarkers currently recommended for using in clinic. } \\
\text { Key results from the AlzBiomarker database based on meta-analysis of decades of research for biomarkers of Alzheimer's disease [11]. Candidate biomarkers are } \\
\text { arranged according to p-values and z scores (standard deviations from means indicating how far away the observed ratio is from the null hypothesis of no difference). } \\
\text { AD: Alzheimer's disease; CSF: Cerebrospinal fluid; NFL: Neurofilament light chain; NSE: Neuron specific enolase; STREM2: Immune receptor expressed on the surface } \\
\text { of microglia. }\end{array}$} \\
\hline
\end{tabular}

\section{Search for circulating AD biomarkers in blood}

Two types of biomarkers can be distinguished in the blood: circulating molecules in plasma/serum and cell-based biomarkers. Recently, most efforts have been concentrating on existing and novel circulating AD biomarkers in the CSF and their verification in blood plasma/serum. The broadly accepted CSF biomarkers: A $\beta 42$ and $\tau$ (total and phosphorylated), as well as new candidate AD biomarkers in the CSF, were analyzed in plasma/serum in multiple studies and cohorts (reviewed in [10-12]). Unfortunately, according to the meta-analysis data of all such studies conducted so far, none of these markers proved to significantly differentiate the blood of $\mathrm{AD}$ patients from controls (Table 2) [11,12].

\section{Search for cell-based biomarkers in the blood based on AD pathology hypotheses}

Facing failure of $A \beta 42$ and other circulating biomarkers in blood plasma/serum, recently increasing attention is turned to the search for cell-based AD biomarkers in the blood. The high diagnostic potential of putative cell-based $\mathrm{AD}$ biomarkers is reflected not only in basic research, but also in the interests of several commercial ventures.

This review summarizes the search results for $\mathrm{AD}$ molecular biomarkers in the most broadly analyzed blood cells: platelets, lymphocytes and erythrocytes. Our approach was to review the data from the perspective of AD pathology hypotheses that were verified in these cells rather than high-throughput screenings. Currently such studies are mostly at the stage of testing hypotheses for proof of principle, therefore the data are less quantitative in terms of diagnostic parameters and does not allow for the classification of probable versus possible biomarkers. However, the most important advantage of such a hypothesis-driven approach is the possibility that these biomarkers could indicate biologically relevant pathways and processes contributing to the complex pathology of individual patients. Therefore, they could inform in the future about possible individualized therapies in $\mathrm{AD}$ patients and about risk factors in those with preclinical AD.

While this review focuses on studies searching for potential AD biomarkers in blood cells as the most easily accessible cell types, such research in $\mathrm{AD}$ is a part of cell-based approaches involving other cell types such as skin fibroblasts and Buccal cells (reviewed in $[10,13,14])$. 


\section{Hypotheses of AD molecular mechanism}

The lack of effective $\mathrm{AD}$ treatments stems mainly from the incomplete understanding of $\mathrm{AD}$ causes. Currently, there are several hypotheses which try to explain the molecular mechanisms of $\mathrm{AD}$ pathogenesis. Among these the amyloid cascade hypothesis is the best known and it cites $\mathrm{A} \beta$ deposits in the AD brain [15]. According to this hypothesis, a cause of AD is the abnormal metabolism of APP involving presenilins and resulting in the overproduction of the neurotoxic peptide $A \beta$ and its oligomerization. $A \beta$ oligomers trigger a cascade of events that leads to synapse dysfunction, neuronal death and the hyperphosphorylation of $\tau$ protein [15]. The $A \beta$ cascade hypothesis has laid the groundwork for the search for anti-AD drugs. To date, however, all of the clinical trials based on this hypothesis have failed. One might begin to question why. Literature presents us with two main responses to this question. One point of view still accepts that the primary trigger of AD pathology is the amyloid. However, a new version of the amyloid hypothesis suggests soluble oligomers initiate the disease process much earlier, before full symptoms can develop [15]. What this means for new therapeutic strategies based on this concept is that it appears necessary to intervene much earlier, long before the development of full AD symptoms. The other response among researchers is that the origins of $\mathrm{AD}$ should be fully reviewed and reconsidered. For example, Herrup recently questioned the $A \beta$ cascade [16], proposing that even though $A \beta$ plaques and $A D$ are highly correlated, there are earlier molecular mechanisms preceding $\mathrm{A} \beta$ production, leading to $\mathrm{AD}$. Moreover, recent studies in humans and in animal models provided evidence that the mechanisms leading to $\mathrm{AD}$ are heterogeneous $[17,18]$. For example, genome wide association studies demonstrated multiple genetic risk factors in AD, other than presenilins or APP [18]. Therefore, in recent years there has been a growing interest in other hypotheses of $\mathrm{AD}$ pathogenesis. It is suggested that causal factors in $\mathrm{AD}$ include oxidative and mitochondrial stress, dysfunctions of cell cycle regulatory proteins, induction of inflammatory cascades as well as impaired glucose and lipid metabolism, and calcium homeostasis. While there is still no clear understanding of the relationship of these processes with $\mathrm{A} \beta$ toxicity and $\mathrm{AD}$ progression phases, the research concerning these molecular changes in $\mathrm{AD}$ has highlighted the complexity of the $\mathrm{AD}$ pathomechanism. It has also highlighted the possibility of deciphering the $\mathrm{AD}$ molecular signature for identifying novel therapeutic targets and an improved AD diagnosis based on novel biomarkers. Further, we review the search for AD biomarkers in blood cells focusing on such main hypotheses of $\mathrm{AD}$ pathogenesis as amyloid cascade, oxidative and mitochondrial stress, aberrant cell cycle and inflammation, as shown schematically in Figure 1, where molecular changes in neurons (Figure 1A) are compared with those identified in platelets, lymphocytes and red blood cells (Figure 1B \& D).

\section{Prospective AD biomarkers in platelets}

\section{Amyloid cascade in platelets}

Platelets are anucleated cells crucial for hemostasis and clot formation at sites of vascular injury. For decades, platelets have been considered a good model to study the pathophysiology of $\mathrm{AD}$ and a promising source of $\mathrm{AD}$ biomarkers, because platelets display all the enzymes of an amyloidogenic pathway and generate $A \beta$ peptides (Figure 1B) [19]. It has been suggested that full-length APP acts as a receptor on the platelet surface and also appears to be crucial in the regulation of intracellular $\mathrm{Ca}^{2+}$ concentration [20]. The proteolytic cleavage of platelet APP appear to occur both on the platelet cell membrane and within the intracellular compartments, and $A \beta$ can be secreted upon platelet activation [21]. In addition to high quantities of APP and A $\beta$ peptides, platelets store and release neurotransmitters, such as serotonin, glutamate and dopamine, and express some neuronal receptors, such as NMDAR [22,23]. Thus, platelets share some properties with neurons, and afford a platform for analyzing the interplay between $A \beta$ and other components that are important for neuronal functionality.

Studies of AD platelets showed alterations in APP processing, membrane fluidity, cholesterol levels, serotonin uptake and intracellular $\mathrm{Ca}^{2+}$ levels. In $\mathrm{AD}$ platelets from $\mathrm{AD}$ patients, changes in the ratio between different isoforms of APP were reported, which correlated with a cognitive decline [24,25]. While alternative splicing of the APP gene can generate at least ten different mRNAs, the isoform predominantly expressed in neuronal tissues is APP695, whereas in non-neuronal cells the most abundant isoforms are APP751 and APP770 [24]. Differences in isoform composition between neurons and platelets raise some disbeliefs about whether functional studies of platelet APP can be directly related to the role of APP in neurons. Nevertheless, associations between early stages of AD and reductions of the platelet APP isoform ratio were demonstrated, proving that the APP isoform ratio in platelets may act as an easily tested early biomarker of $\mathrm{AD}$ [24].

The proteolytic processing of APP in nonamyloidogenic pathway mediated by $\alpha$-secretase (ADAM10) produces a secreted ectodomain fragment $(\mathrm{sAPP} \alpha)$ that has neuroprotective and neurotrophic properties. A reduced level of ADAM10 is observed in platelets obtained from $\mathrm{AD}$ and MCI patients compared with age-matched controls [25]. 
Furthermore, a qualitatively and quantitatively similar decrease in sAPP $\alpha$ is present both in thrombin-activated platelets and the CSF, thus suggesting that alterations of APP processing might occur both in the neuronal compartment and peripheral cells [25].

Another study also suggested APP as potential platelet AD biomarker, but this time on the platelet surface, related to coated-platelets [26]. Coated platelets (formerly known as COAT-platelets), represent a subpopulation of cells

(A)
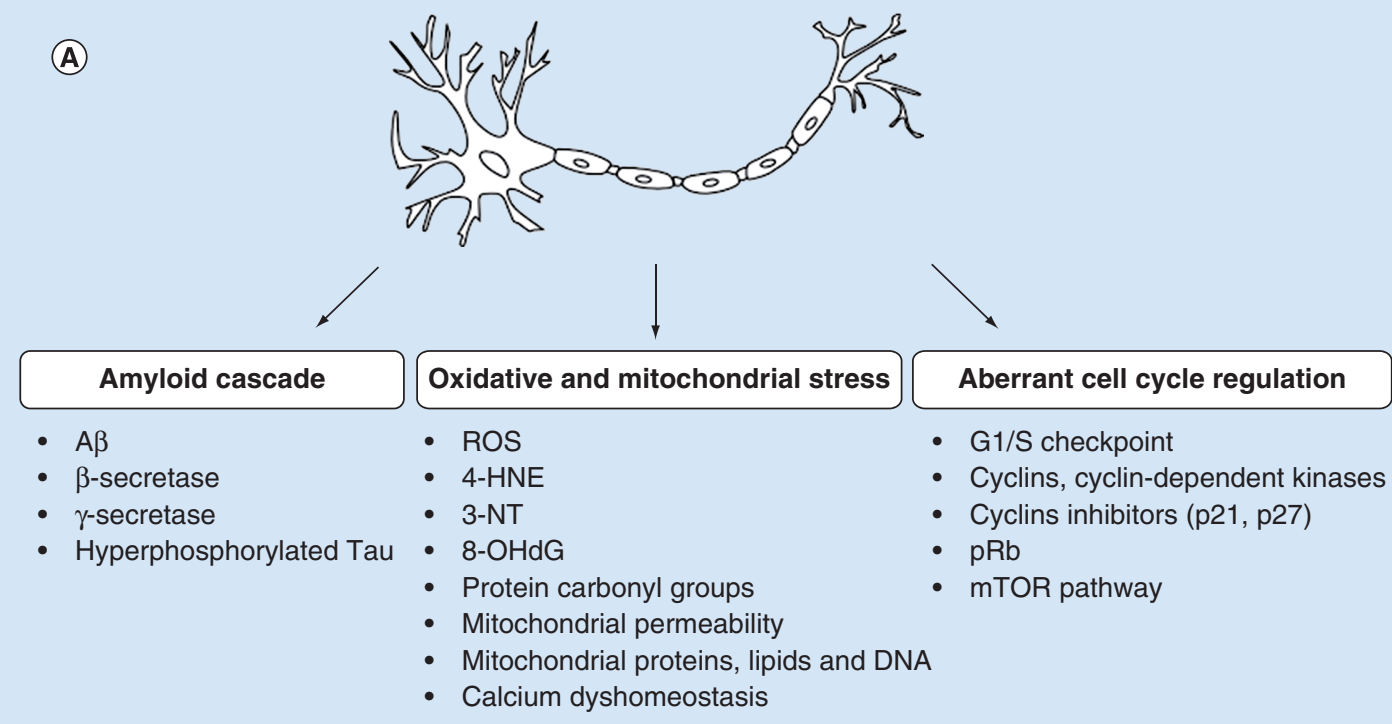

(B)
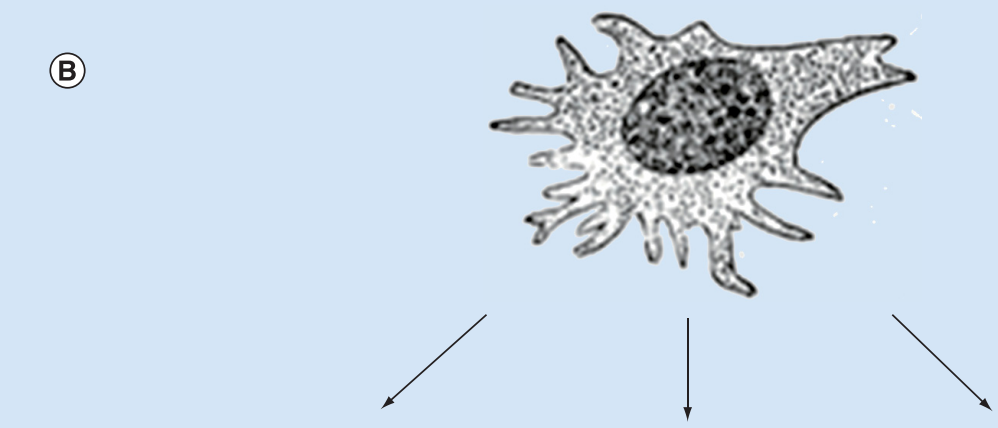

\section{Amyloidogenic pathway}

\section{Oxidative and mitochondrial stress}

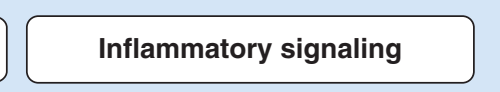

- APP isoforms

- sAPP $\alpha$ ADAM10

- ROS

- Mitochondrial electron transport chain

- $\operatorname{cox} 2$

- Tau

- GSK3ß

- Coenzyme Q10

- NOS

- Nitric oxide and peroxynitrite

- PLA2

- Secreted A $\beta$

- Platelet activation

- Intracellular $\left[\mathrm{Ca}^{2+}\right]$

- Lipid peroxidation

- $\mathrm{Na}+, \mathrm{K}+-\mathrm{ATPase}$

- PLC

- MOA

- Apoptosis (caspases, Bad, Bax, Bcl2)

- Cholesterol and GM1 in lipid rafts

- Serotonin

Figure 1. Main molecules and processes altered in Alzheimer disease cells.

(A) neurons, (B) platelets, (C) lymphocytes, (D) red blood cells. Changes in levels and/or activities of molecules in cells from Alzheimer's disease patients comparing to controls are shown. 
(C)

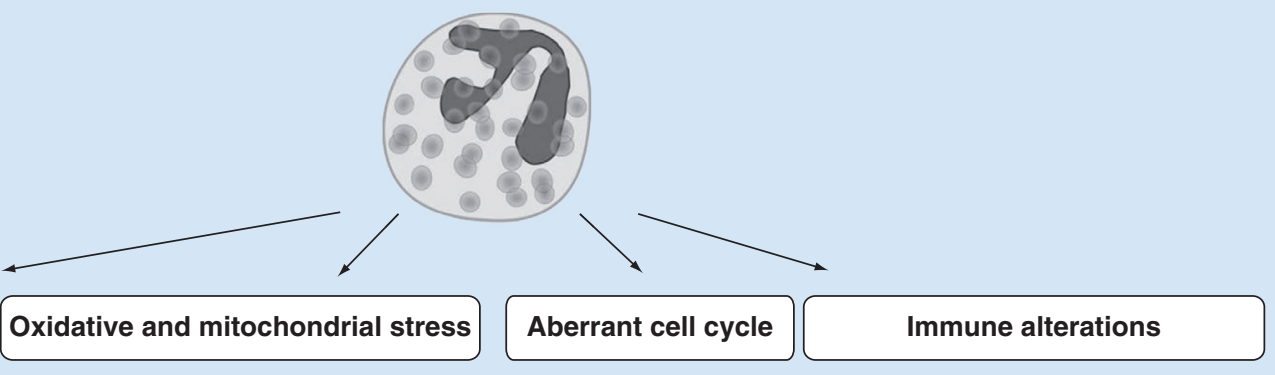

Amyloidogenic pathway

- ROS

- 4-HNE

- B cells proliferation rate

- APP isoforms

- Protein carbonyl groups

- G1/S checkpoint

- Activation of T-cells (CD25)

- 3-NT

- Cyclin D1

- Chemokine receptors (CCR5, CCR6)

- 8-OHdG

- p21

- Cytokine production

- GSH/GSSG

- $\mathrm{pRb}$

- Profile of T cell subpopulation

- SOD

- NF-кB pathway

- TR

- mTOR pathway

- $\mathrm{HO}-1$

- NOS2

- HSP60, HSP72

- Mitochondrial electron transport chain

- Mutation rate in mitochondrial DNA

- Conformationally altered p53

(D)

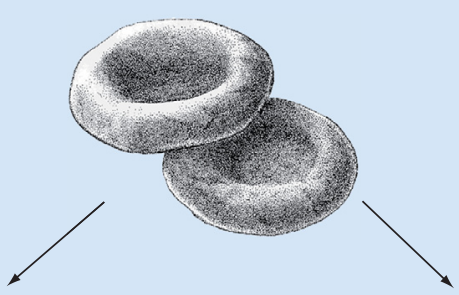

\section{Amyloidogenic pathway}

- $A \beta 40$ and $A \beta 42$

Oxidative stress and erythrocyte physiology

- Morphological changes induced by $A \beta$ binding

- ROS

- Phospholipid hydroperoxides PLOOH

- Hydrogen peroxide

- Glutathione transferase

- GSH/GSSG

- 2,3-diphosphoglycerate

- Glycolytic enzymes

- Caspase 3

- PKC

- External AchE

- Long-chain polyunsaturated fatty acids (OMEGA-3)

Figure 1. Main molecules and processes altered in Alzheimer disease cells (cont.).

(A) neurons, (B) platelets, (C) lymphocytes, (D) red blood cells. Changes in levels and/or activities of molecules in cells from Alzheimer's disease patients comparing to controls are shown.

observed after dual agonist stimulation of platelets with collagen and thrombin. This class of platelets retains on its surface high levels of several procoagulant proteins, including fibrinogen, fibronectin and thrombospondin. Coatedplatelets also express surface phosphatidylserine and retain full-length APP on their surface upon activation [26]. The number of coated platelets with full-length APP is contrarily correlated with the severity of the cognitive decline, in other words higher levels are found at earlier stages of the dementing process in MCI patients, and higher counts of coated platelets at baseline predicted a more severe cognitive deterioration over time [26]. 
Research involving AD platelets concerns also glycogen synthase kinase (GSK3 $\beta$ ), a serine-threonine kinase involved in the hyperphosphorylation of $\tau$ in $A D$ and in regulation of crucial intracellular signaling pathways, including cell cycle and apoptosis [27]. The activation of GSK3 $\beta$ has been linked both with the pathology of $\tau$ and $\mathrm{A} \beta$ as GSK3 $\beta$ promotes amyloidogenic cleavage of APP [28]. GSK3 $\beta$ and $\tau$ protein expression in platelets is higher compared with other blood cells. The increased platelet GSK3 $\beta$ activity was reported in patients in early $\mathrm{AD}$ stages [29]. The definite role of platelet GSK3 $\beta$ is still unclear, but there is evidence of its involvement in the regulation of platelet activation and $\tau$ forms in $\mathrm{AD}$ [28-30]. The potential of molecular changes related to $\tau$ and amyloid pathology in platelets as diagnostic biomarkers is promising and awaits further investigation.

\section{Mitochondrial \& oxidative stress in platelets}

Another line of research investigated mitochondrial and oxidative stress in AD platelets (Figure 1B). Fišar et al. demonstrated that analyses of the respiratory rates of intact platelets, complex IV activity and the capacity of the electron transport chain in permeabilized platelets may enable the characterization of mitochondrial dysfunctions in the initial AD stage [31].

Mitochondrial stress is often linked with apoptosis and indeed, a higher level of apoptosis was demonstrated in $\mathrm{AD}$ platelets [32], in agreement with altered apoptosis reported in other blood cells [33]. Platelet levels of caspase-3, caspase-9, Bad and Bax proved to be significantly increased in $\mathrm{AD}$ and amnestic MCI. The antiapoptotic protein $\mathrm{Bcl} 2$ increased in amnestic MCI, while it decreased in $\mathrm{AD}$. It thus seems that abnormal apoptosis may appear in the early stages of $\mathrm{AD}$, and the ratio between pro- and antiapoptotic protein levels partially determines the susceptibility of platelets to a death signal. Therefore, platelets may also be a good model to study apoptotic pathways during $\mathrm{AD}$ progression [32].

$\mathrm{AD}$ platelets exhibit also several markers and components of oxidative stress, including the increased lipid peroxidation, $\mathrm{Na}+, \mathrm{K}+$-ATPase activity, increased production of nitric oxide and peroxynitrite, and the compromised activity of key enzymes in platelet signal transduction in the cell membrane (phospholipases), several enzymes in the cytosol (e.g., monoamine oxidase, MOA), and in mitochondria (nitric oxide synthase [NOS]) [34].

An important question that emerges is the relationship between mitochondrial stress and the amyloid cascade in $\mathrm{AD}$. This is still unclear, as is the sequence of events in $\mathrm{AD}$ pathogenesis. Platelets seem to represent a proper model for such studies and sustain a promising source for molecular AD biomarkers.

\section{Inflammatory signaling in platelets}

Under pathological conditions, platelets play an important role in thrombus formation and thrombocytopenia, and also in inflammation, which are all known risk factors for $\mathrm{AD}$ [35,36]. AD's familial mutations result in a hyperactivated state of circulating platelets, evident with the disease's progression [37]. The most prevalent inflammatory signaling molecules secreted by platelets include chemokines, interleukins and adhesive proteins; platelets also secrete prostaglandins produced by the enzymes COX-1 and -2 [38]. An example of platelet markers of the inflammatory response associated with $\mathrm{AD}$ is $\mathrm{COX} 2$ enzyme, elevated in platelets of $\mathrm{AD}$ and $\mathrm{MCI}$ patients [39]. Decreased activity of platelet phospholipase A2 (PLA2), another regulator of the inflammatory response, proved to predict conversion from MCI to AD [40].

\section{Platelet levels of lipids \& serotonin}

Among promising AD biomarkers in platelets are lipids [41] and neurotransmitter serotonin (5-HT) [42]. The cholesterol and ganglioside GM1 content of lipid rafts from platelets was found significantly higher in AD than in aged-matched control subjects [41]. In turn, 5-HT plays a significant role in cognition and a combination of disturbances in cholinergic and serotonergic functions may play a role in cognitive impairment in AD. Discovering that platelets' dense granules are the major storage site for serotonin 5-HT in the blood, similar to its vesicular storage in neurons, triggered the idea of using platelets as an experimental system in $\mathrm{AD}$. AD patients showed significantly reduced platelet 5-HT levels; furthermore lower 5-HT content in patients with Subjective Cognitive Impairment was associated with a higher AD-CSF biomarker burden [42]. Thus, platelets 5-HT seem to be a good candidate biomarker for early AD.

\section{Contribution of platelets to systemic changes in $A D$}

Platelets have also been studied as a peripheral cell type that can actively contribute to systemic $\mathrm{AD}$ changes, mainly to vascular dysfunctions that are often associated with $\mathrm{AD}[43,44]$. Platelets provide over $90 \%$ of blood $\mathrm{A} \beta$. It is 
known that during thrombosis, platelets are concentrated in clots and activated, and that activated platelets release $A \beta$. Enhanced release of $A \beta$ during thrombosis suggests an additional source of $A \beta$ in the brains of $A D$ patients with frequent micro-thrombosis events occurring in their brains [45]. This possibility is consistent with findings in mice models and with highly conserved pattern of $A \beta$ peptides that are known to be released by platelets in the CSF $[43,45]$. Some authors suggested an inverse pathway, in other words that circulating $A \beta$ derives from the CNS, crosses the blood-CSF barrier, and can be absorbed by platelets and other blood cells. Regardless of its origin, $\mathrm{A} \beta$ produced by platelets or derived from the brain may affect the platelets. Therefore, the role of extracellular $A \beta$ in platelet physiology was investigated. $A \beta$ was shown to activate platelets and trigger platelet aggregation by stimulating intracellular signaling pathways that are involved in phospholipase $\mathrm{C}$ phosphorylation, protein kinase $\mathrm{C}(\mathrm{PKC})$ activation and increased intracellular $\left[\mathrm{Ca}^{2+}\right]$, resulting in increased $\mathrm{A} \beta$ secretion. These findings raise the possibility that $A \beta$ activation may initiate a mutual cycle of platelet activation and $A \beta$ release, which may play a role in $A \beta$ upregulation in the periphery and in the CSF, further leading to the development of cerebral amyloid angiopathy [43-45].

In summary, platelets have been studied as both a cellular model to investigate $\mathrm{A} \beta$-induced responses, and a peripheral cell type that actively contribute to $\mathrm{AD}$ pathogenesis. Main molecules and platelet functions that can be applied as potential AD biomarkers are shown in Figure 1B.

\section{Prospective AD biomarkers in lymphocytes}

The use of lymphocytes in $\mathrm{AD}$ research consists of analyzing lymphocytes isolated directly from AD patients' blood, $\mathrm{B}$ and $\mathrm{T}$ cells separately or as a mixed lymphocyte fraction, and B-lymphocytes immortalized with the Epstein-Barr virus, in other words proliferating B-lymphoblasts [46]. B-lymphoblasts were especially valuable as a source of higher quantities of research material and as a model of activated, proliferating B cells for cell cycle studies.

AD lymphocytes differentiate from healthy controls by changes in APP isoform levels, increased mitochondrial and oxidative stress, changes in cell cycle regulation and in molecular alterations related to immune functions (Figure 1C).

\section{Amyloid cascade components in lymphocytes}

AD lymphocytes express three isoforms of APP, namely APP695, APP751 and APP770. The ratio of APP751:APP770 mRNA levels is lower in AD lymphocytes compared with controls [46]. To date, no study has attempted to verify $A \beta$ production in lymphocytes. BACE1 expression was found in lymphocytes but no nicastrin expression was reported [47], and this indicates low probability of $A \beta$ peptides production by the $\gamma$ secretase and low potential of amyloid cascade components as $\mathrm{AD}$ biomarkers in lymphocytes.

\section{Oxidative \& mitochondrial stress in lymphocytes}

Increasing evidence shows elevated levels of oxidative stress markers in nucleic acids, carbohydrates, lipids and proteins in $\mathrm{AD}$ patients' brains, as well as in the periphery. The results showing oxidative and mitochondrial stress markers in AD lymphocytes were recently reviewed in [46]. Reactive oxygen species (ROS) are inclined to attack the lipid bilayers' polyunsaturated fatty acids, which leads to reactive aldehydes forming, among which one of the most toxic is 4-hydroxy-2-nonenal. When proteins react with 4-hydroxy-2-nonenal, it can lead to stable covalent adducts forming with cysteine, lysine and histidine residues. This aldehyde and protein oxidation markers such as protein carbonyls and 3-nitro tyrosine were significantly elevated in $\mathrm{AD}$ lymphocytes and showed a negative correlation with MMSE. Moreover, the superoxide dismutase activity was reduced in AD lymphocytes, showing an impairment in the antioxidant enzymes response [48]. Furthermore, elevated protein levels and activities of NOS2, HO-1, HSP60, HSP72 and thioredoxin reductase were found, which is to be expected when occurring in an oxidizing environment [49].

One of the main sources of oxidative stress are mitochondria as they utilize oxygen for energy production. Regulation of cell death seems to be intimately linked to their role as the major intracellular source of ROS, which are mainly generated at Complex I and III of the respiratory chain mitochondrial proteins' oxidative modification causes the mitochondria to increasingly leak superoxide radicals, which may result in an increased production of reactive nitrogen and oxygen species that may consecutively impact cellular functions and death lipid and protein oxidation in mitochondria are considered by several research groups to be potential biomarkers for AD [46,48]. The mitochondrial respiration capacity was determined as oxidative phosphorylation (OXPHOS) and delineated the whole electron transport chain of the four mitochondrial enzymes (complexes I-IV). AD patients exhibited a 
reduced endogenous basal rate of respiration (state1), as well as significant impairment of total OXPHOS capacity. In addition, the respiratory control ratio: coupled OXPHOS capacity (state3) divided by leak flux (state4), which is an indicator of the mitochondria's coupling state, decidedly dropped in AD lymphocytes. This confirms that in $\mathrm{AD}$ cells there exists a functional deficit of oxygen consumption, and that it can consequently prompt failure of cellular energetic maintenance.

Mitochondria extracted from AD lymphocytes also showed raised levels of protein oxidation markers: $3 \mathrm{NT}$ and protein carbonyls, as well as protein-bound HNE, a lipid peroxidation marker. This is in accordance with increased glutathione disulfide levels and lowered plasma levels of glutathione GSH observed in AD lymphocytes [46]. Furthermore, recent data showed that increased susceptibility to oxidative death of lymphocytes from AD patients correlates with dementia severity [50].

An additional molecular change connected to mitochondrial oxidative stress in the brains and lymphoblastoid cells of $\mathrm{AD}$ patients is an increased frequency of DNA mutation in mitochondrial DNA (mtDNA) [51]. Numerous such mutations were found in locations of known mtDNA replication and transcription regulatory elements, thus leading to reduced transcript levels of crucial mitochondrial proteins that were deleterious to mitochondrial functions. The absence of protective proteins such as histones, the shortage of an efficient DNA repair system, and the close proximity to the ROS generation source, makes mtDNA very sensitive to an ROS attack.

Overall, the studies all emphasize the systemic nature of oxidative stress in AD. Elevated ROS levels, altered levels of antioxidant enzymes, elevated hydroxyl radical-induced DNA oxidation, and increased mitochondrial sensitivity were found in $\mathrm{AD}$ lymphocytes compared with controls. Increased mitochondrial and oxidative stress markers reflect the pathological changes of AD brains. Furthermore, considerable mitochondrial dysfunction has been found in MCI lymphocytes, without any $\tau$ or $A \beta$ pathology (reviewed in [46]). These data confirm that oxidative stress markers may potentially become markers for early AD diagnosis (Figure $1 \mathrm{C}$ ).

\section{Dysregulated cell cycle in lymphocytes}

In fully differentiated neurons cell cycle is constantly blocked. Surprisingly, multiple recent studies suggest that cell cycle proteins' aberrant re-expression and the reentry of the cell cycle may represent a pathological feature of mature AD neurons [52]. Research points out that rather than being a consequence of neurodegeneration, cell cycle re-entry is its cause, and consequently cell cycle regulatory proteins of G1/S phase checkpoint may develop into early AD biomarkers [46,53,54].

Cell cycle disturbances also occur in AD lymphocytes, as summarized in Figure 1C. A few groups demonstrated a specific failure of the G1/S transition checkpoint in lymphocytes of $\mathrm{AD}$ patients (reviewed in [46]). This supports the $\mathrm{AD}$ cell cycle hypothesis of mitosis failure and validates using peripheral blood cells to study AD pathogenesis' molecular basis and search for AD biomarkers.

A potential AD biomarker in lymphocytes seems to be the 21 protein, one of the most significant regulators of the G1/S checkpoint and the mediator of cell cycle arrest in the G1 phase [55]. In SAD lymphocytes an impaired progression of the G1/S phase occurs, consistent with p21's main change and its known function in arresting G1 [17]. Experiments with serum withdrawal also exhibited elevation of p21 level in the cytosol and changes of E2F and NF- $\mathrm{KB}$ transcription factors' activity [56]. The reduced NF- $\mathrm{KB}$ activation in $\mathrm{AD}$ cells seems to protect them from death induced by the loss of trophic factors.

A line of research focused on the p53 protein, a tumor suppressor well known for its role in cell cycle arrest, DNA repair and apoptosis. 553 is crucial for the induction of genes that lead to G1 arrest following DNA damage, enabling DNA repair. AD lymphocytes express an anomalous and detectable conformational state of p 53 defined as a 'mutant like' conformation [57]. p53 adopts at least two tertiary structures in vivo, known as conformations: wild-type functionally active and mutant-type functionally inactive. In normal cells wild-type, p 53 is constitutively expressed at low levels and short-lived. When exposed to stress, wild-type p 53 activates, stabilizes and is imported into the nucleus, where it acts as the transcription factor of several genes which are responsible, among others, for G1 cell cycle arrest and DNA repair [58]. Conformational p53 mutants cannot employ these functions and lose their G1/S transition suppressor potential. Therefore, identifying increased levels of $\mathrm{p} 53$ conformational mutants in $\mathrm{AD}$ lymphocytes implies that conformational alterations of $\mathrm{p} 53$ may be accountable for the dysfunction of the G1/S checkpoint in AD.

It was also analyzed whether a diagnostic biomarker for $\mathrm{AD}$ could be based on differences in the proliferation activity of peripheral blood lymphocytes. Using univariate models in AD subjects eleven CD expression markers were found to be significantly changed compared with a mixed pool of HC and PD subjects [59]. The results suggest 
that both for the pathomechanism of $\mathrm{AD}$ and the development of $\mathrm{AD}$ diagnostic markers, a systemic failure of cellular proliferation control is critically important.

Cell cycle dysregulation also appears in lymphocytes from other neurodegenerative disorders such as frontotemporal lobar degeneration (FTLD-TDP) [60], but not in amyotrophic lateral sclerosis [61]. These data suggest that in many neurodegenerative diseases the dysregulation of the cell cycle is a convergent point but the particular signaling pathways mediating dysfunction of the cell cycle is different $[37,60]$. Thus, it is important to determine the disease-specific, unique molecular signature of the changes in cell cycle-related proteins in $\mathrm{AD}$ cells compared with cells in other neurodegenerative diseases.

\section{Immune alterations in lymphocytes}

Several groups have attempted to clarify if oxidative and mitochondrial stress, as well as aberrant cell cycle could induce such physiological changes in AD lymphocytes that would affect their immune function. A clear picture has yet to be defined. The following question is the exact function of the peripheral immune system in AD.

In the $\mathrm{AD}$ brain, the deposition of $\mathrm{A} \beta$ plaques and NFTs leads to the activation of glia and gliosis, considered to represent an important histopathological $\mathrm{AD}$ lesion [62]. Additionally to the activation of microglia and astrocytes, which are resident brain cells, this local innate immunity entails various inflammation-related proteins, including acute-phase proteins, complement factors, chemokines and proinflammatory cytokines [62]. Moreover, increasing evidence suggests that peripheral immune cells play a role in the brain's pathological events [63].

The skull, CSF and blood-brain barrier (BBB) all protect the human brain. The BBB is disturbed already in early $A D$ [64]. The breakdown of the $B B B$ in $A D$ increases the amounts of $A \beta$ and other toxins in the brain. Monocytes can cross the $\mathrm{BBB}$ and, upon infiltration, take part in the phagocytosis of $\mathrm{A} \beta$ plaques as macrophages. In addition to macrophages, T-lymphocytes are also activated in $\mathrm{AD}$ patients, crossing the $\mathrm{BBB}$ and infiltrating the brain. T-cell migration through the BBB appears to be related to the upregulation of CC chemokine receptors, such as receptor 5 (CCR5). Furthermore, in AD patients the percentage of cells expressing a marker of acute T-cell activation CD25, as well as percentages of CCR6 ${ }^{+}$cells, were found to be higher than in age-matched controls. Unbalanced cytokine production and altered T-lymphocyte subset profile indicate that T-lymphocytes have an active part in $\mathrm{AD}$ pathology.

B cells, in opposition to T-lymphocytes, remain in peripheral circulation and do not infiltrate the BBB. The contribution of $\mathrm{B}$ cells in AD pathology has therefore been underestimated. Presently, a growing body of data indicates that perturbations in B-cell immunoreactivity are also a feature of AD pathology [46].

Overall, molecular alterations in blood lymphocytes correlate with disturbances in the AD immune system. An increasing amount of evidence suggests a role of peripheral immune cells in the pathological events that occur in the brain.

\section{Potential AD biomarkers in red blood cells}

Erythrocytes red blood cells (RBCs) are simple and unique cells due to the lack of a cell nucleus and consequently nuclear genes, ribosomes and protein synthesis. Because the erythrocyte represents the body's oxygen carrier, its redox and metabolic status is extremely important for the functioning and regulation of oxygen affinity to hemoglobin, which is determined by a number of metabolites within the erythrocyte. All tissues are dependent on RBC function, especially neurons which use $20 \%$ of the total oxygen consumed.

\section{$A \beta$-induced morphological changes in erythrocytes}

The number of studies on red blood cells and $A D$ are based on the interaction of $\mathrm{RBC}$ and $\mathrm{A} \beta$ in the blood stream and its physiological implications. Potential contribution of impaired oxygen delivery to the brain, causing associated neuronal $\mathrm{AD}$ dysfunction, has been considered an important factor in $\mathrm{AD}$ [65]. Oxygen delivery to the brain requires that red blood cells deform to pass through the narrow capillaries that supply oxygen to the brain. Vascular changes associated with amyloidosis are expected to directly impair blood flow. The vascular hypothesis of $\mathrm{AD}$ considers cerebral hypoperfusion as a primary trigger for neuronal dysfunction [65].

As, it has been mentioned earlier, in addition to $A \beta$ peptide deposition in neurons, it has been found in blood plasma, at nanomolar concentrations [66]. $\mathrm{A} \beta 40$ and $\mathrm{A} \beta 42$ were detected also in RBCs where their levels were found to be about 8- and 14-times higher than those in plasma, respectively [67]. Exposure of RBCs exposed to A $\beta$ on the luminal surface of cerebral microvessels induces the erythrocytes' adhesion to endothelial cells and affects endothelial functionality [68]. In addition, bounding of $A \beta$ to RBCs impairs the capacity of RBCs for delivering 
oxygen thereby facilitating AD [65-69]. It was shown that oxidative stress and ROS production in RBC membranes was induced particularly by copper-bound $A \beta$ [65]. This ROS pool could leak out of the RBC and damage nearby cells or tissues. Recent data indicated that $98 \%$ of AD peripheral RBCs is amyloid binding-positive, while only $38 \%$ of such RBCs were found in healthy controls. Four modified morphologies of RBCs triggered by A $\beta$ binding were suggested to indicate $\mathrm{AD}$ progression [69]. In agreement with these notions, Down syndrome patients with elevated $\mathrm{A} \beta$ levels in blood plasma were reported to have a greater risk of developing $\mathrm{AD}$ [70,71].

\section{Oxidative stress \& altered energy production in RBCs}

$\mathrm{A} \beta$ binding induces oxidative injury to RBCs and causes the accumulation of phospholipid hydroperoxides, a specific marker for RBC membrane oxidative injury [72]. Moreover, an inverse relationship was seen between RBC concentrations of phospholipid hydroperoxides and carotenoids in AD patients [72]. Erythrocytes do not have sufficient mechanisms to repair oxidized lipids in their membranes, which leads to the accumulation of these products in the membranes as well as inside the cell during the erythrocyte's lifespan. Fluorescence spectroscopy studies revealed the significantly increased level of lipid peroxidation specific products in erythrocytes and plasma of $\mathrm{AD}$ dementia and MCI patients [73,74]. These altered molecules could potentially be applied as biomarkers for detection of early $\mathrm{AD}$.

In erythrocytes, similarly as in neurons, an increase of $A \beta$ toxicity is mediated by its binding with dimeric acetylcholinesterase AChE forms located on the RBCs external face [75]. External acetylcholinesterase conformational status is dependent on NO metabolism, involving PKC activity [75]. It was demonstrated that in RBCs PKC, in consideration of its known crucial role in RBC signalling, could play a key role in A $\beta$-induced oxidative imbalance [75].

An important aspect in RBC functioning is energy production, related closely with redox status. Indeed, in $\mathrm{AD}$ erythrocytes increased oxidative stress is manifested in the elevated concentrations of hydrogen peroxide and decreased glutathione/glutathione disulfide ratio and glutathione transferase activity [76]. These changes are associated with reductions in ATP levels, adenine nucleotide pool size and adenylate energy charge [76]. In particular, 2,3-diphosphoglycerate levels were decreased in AD patients. Moreover, erythrocytes from $\mathrm{AD}$ patients showed increased activity of four glycolytic enzymes (phosphofructokinase, hexokinase, bisphosphoglycerate phosphatase and bisphosphoglycerate mutase).

There have also been several of studies on the fatty acids composition of the RBC membrane in $\mathrm{AD}$ and in MCI. $\mathrm{AD}$ and $\mathrm{MCI}$ patients exhibit lower levels of long-chain polyunsaturated fatty acids (PUFA) of Omega-3 series in RBC membranes compared with healthy controls [77]. Moreover, lower erythrocytes levels of Omega-3 PUFA, eicosapentaenoic acid and docosahexaenoic acid, were shown to be associated with smaller brain volumes and a 'vascular' pattern of cognitive impairment [78]. The findings in $\mathrm{AD}$ erythrocytes support the erythrocytic/vascular hypothesis of $\mathrm{AD}$ and contribute to a molecular signature of $\mathrm{AD}$ in $\mathrm{RBCs}$.

\section{Conclusion \& future perspective}

$\mathrm{AD}$ is commonly regarded as a brain disease, nevertheless, increasing data show that $\mathrm{AD}$ also exhibits a systemic presentation. The reviewed data document molecular and cellular changes in AD blood cells, in early and advanced $\mathrm{AD}$. Currently this research is mostly at the stage of testing for proof of principle, nevertheless lays the ground for the future development of a panel of different molecules in blood cells comprising a molecular signature of AD. If peripheral blood cells were used, the detection of AD could be carried out employing straightforward, noninvasive and inexpensive diagnostic assays, compared with currently administered tests that involve the withdrawal of CSF or brain imaging. Importantly, it seems that combining several blood-based biomarkers might allow for the exact stratification of AD stages and forms, informing on contributing complex pathomechanisms in AD patients and possible individualized therapies. Future diagnostics will require a correlation of stage-specific blood-based biochemical biomarkers with brain imaging and neuropsychological tests.

\section{Acknowledegments}

The authors thank A Piotrowska for her excellent editorial help.

Financial \& competing interests disclosure

Creation of this work has been supported by the European Union's Horizon 2020 research and innovation program under the Marie Sklodowska-Curie grant agreement no 665735 (Bio4Med) and by the funding from Polish Ministry of Science and Higher Education 
within 2016-2020 funds for the implementation of international projects (agreement no 3548/H2020/COFUND/2016/2) to U Wojda. The work was carried out with the use of CePT infrastructure financed by the European Union - The European Regional Development Fund within the Operational Program 'Innovative Economy' for 2007-2013. The authors have no other relevant affiliations or financial involvement with any organization or entity with a financial interest in or financial conflict with the subject matter or materials discussed in the manuscript apart from those disclosed.

No writing assistance was utilized in the production of this manuscript.

\section{Executive summary}

\section{Background}

- Alzheimer's disease (AD) is one of the major unmet medical and economic problems of the modern world. Improving the effectiveness of $A D$ therapies is strictly contingent on early diagnosis.

$A D$ diagnostics \& demand for novel, improved biomarkers

- Progress in early $A D$ diagnostics requires identifying $A D$ biomarkers, which will allow not only to detect major hallmarks of $A D$ such as $A \beta$ and $\tau$, but will report the type of contributing complex pathomechanisms in patients with clinical $A D$ and inform about risk factors for progression to dementia in preclinical $A D$.

- New biomarkers should be more cost-effective and present in easily available diagnostic tissues, such as blood.

Search for circulating AD biomarkers in blood plasma/serum

- Recently, most efforts have been concentrating on circulating biomarkers for early AD in the CSF and their verification in blood plasma/serum. Unfortunately, so far none of the circulating markers in the blood proved to differentiate $A D$ patients from controls.

Search for cell-based biomarkers in the blood based on AD pathology hypotheses

- Facing failure of investigated circulating biomarkers in the blood, increasing attention is turned to the search for AD biomarkers in blood cells.

Hypotheses of AD molecular mechanism

- The main hypothesis known as amyloid cascade assumes that the cause of $A D$ is the overproduction of the $A \beta$ peptide. However, other cellular processes seems to cause and/or contribute to complex AD pathology, including oxidative and mitochondrial stress, dysfunctions of cell cycle regulation, inflammatory cascades and impaired glucose and lipid metabolism.

Prospective AD biomarkers in platelets, lymphocytes \& red blood cells

- Among blood cells, only platelets represent a good model for analyzing the interplay between $A \beta$ and other components important for neuronal functionality.

- Common molecular alterations found in all three blood cell types reflect such main hypotheses of AD pathogenesis as amyloid cascade, oxidative and mitochondrial stress and inflammation. Moreover, proliferating lymphocytes reveal alterations in cell cycle regulation, reflecting the cell cycle hypothesis.

- The molecular alterations affect the physiological functions of blood cells and document the systemic nature of AD.

Summary \& future perspective

- The reviewed data supports the rationale of employing blood cells as a model to study the early mechanism of $A D$ pathogenesis and as a source for novel biomarkers in early AD.

- At this moment, none of the blood cell types seem to be more promising than the others; instead a multicellular panel seems preferable for defining complex AD pathomechanisms.

- The diagnostic parameters of cell-based biomarkers in the blood, such as their specificity and sensitivity, await characterization.

\section{References}

Papers of special note have been highlighted as: • of interest; $\bullet \bullet$ of considerable interest

1. Dementia statistics. www.alz.co.uk/research/statistics

2. Braak H, Braak E, Bohl J. Staging of Alzheimer-related cortical destruction. Eur. Neurol. 33(6), 403-408 (1993).

3. Gelosa G, Brooks DJ. The prognostic value of amyloid imaging. Eur. J. Nucl. Med. Mol. Imaging 39(7), 1207-1219 (2012).

4. Jack CR, Knopman DS, Jagust WJ et al. Tracking pathophysiological processes in Alzheimer's disease: an updated hypothetical model of dynamic biomarkers. Lancet Neurol. 12(2), 207-216 (2013).

-. Key article on dynamic biomarkers in preclinical and clinical Alzheimer's disease (AD).

5. Dubois B, Hampel H, Feldman HH et al. Preclinical Alzheimer's disease: definition, natural history, and diagnostic criteria. Alzheimers Dement. 12(3), 292-323 (2016).

-• Most recent review of main concepts and definitions in preclinical AD. 
6. McKhann GM, Knopman DS, Chertkow $\mathrm{H}$ et al. The diagnosis of dementia due to Alzheimer's disease: recommendations from the National Institute on Aging-Alzheimer's Association workgroups on diagnostic guidelines for Alzheimer's disease. Alzheimers Dement. 7(3), 263-269 (2011).

7. Albert MS, DeKosky ST, Dickson D et al. The diagnosis of mild cognitive impairment due to Alzheimer's disease: recommendations from the National Institute on Aging-Alzheimer's Association workgroups on diagnostic guidelines for Alzheimer's disease. Alzheimers Dement. 7(3), 270-279 (2011).

8. Sperling RA, Aisen PS, Beckett LA et al. Toward defining the preclinical stages of Alzheimer's disease: recommendations from the National Institute on Aging-Alzheimer's Association workgroups on diagnostic guidelines for Alzheimer's disease. Alzheimers Dement. 7(3), 280-292 (2011).

9. Bateman RJ, Xiong C, Benzinger TL et al. Clinical and biomarker changes in dominantly inherited Alzheimer's disease. N. Engl. J. Med. 367(9), 795-804 (2012).

- An important article demonstrating diagnostic ability in preclinical AD.

10. Khan TK. Cell-base Alzheimer's disease biomarkers. In: Biomarkers in Alzheimer's Disease. Academic Press, MA, USA (2016).

11. Alzforum Alzbiomarker Version 2.0 (2017). www.alzforum.org/alzbiomarker

-. Meta-analysis of circulating biomarkers in the cerebrospinal fluid and blood.

12. Olsson B, Lautner R, Andreasson U et al. CSF and blood biomarkers for the diagnosis of Alzheimer's disease: a systematic review and meta-analysis. Lancet Neurol. 15(7), 673-684 (2016).

13. Khan TK, Alkon DL. Peripheral biomarkers of Alzheimer's disease. J. Alzheimers Dis. 44(3), 729-744 (2015).

14. François M, Leifert W, Martins R, Thomas P, Fenech M. Biomarkers of Alzheimer's disease risk in peripheral tissues; focus on buccal cells. Curr. Alzheimer Res. 11(6), 519-531 (2014).

15. Selkoe DJ, Hardy J. The amyloid hypthesis of Alzhimer's disease at 25 years. EMBO Mol. Med. 8(6), 595-608 (2016).

- Presentation of the amyloid cascade hypothesis.

16. Herrup K. The case for rejecting the amyloid cascade hypothesis. Nat. Neurosci. 18(6), 794-799 (2015).

-. Contradictory to amyloid cascade arguments; review of other hypothesies of $\mathrm{AD}$ pathology.

17. Bialopiotrowicz E, Kuzniewska B, Kachamakova-Trojanowska $\mathrm{N}$ et al. Cell cycle regulation distinguishes lymphocytes from sporadic and familial Alzheimer's disease patients. Neurobiol. Aging 32(12), 2319.e13-2319.e26 (2011).

18. Hampel H, Lista S. Have we learnt all we need to know from genetic studies - is genetics over in Alzheimer's disease? Alzheimers Res. Ther. 5(2), 11 (2013).

19. Catricala S, Torti M, Ricevuti G. Alzheimer disease and platelets: how's that relevant. Immun. aging 9(1), 20 (2012).

20. Kniewallner KM, Ehrlich D, Kiefer A, Marksteiner J, Humpel C. Platelets in the Alzheimer's disease brain: do they play a role in cerebral amyloid angiopathy? Curr. Neurovasc. Res. 12(1), 4-14 (2015).

21. Gowert NS, Donner L, Chatterjee M et al. Blood platelets in the progression of Alzheimer's disease. PLoS ONE 9(2), e90523 (2014).

22. Milovanovic M, Eriksson K, Winblad B et al. Alzheimer and platelets: low-density platelet populations reveal increased serotonin content in Alzheimer type dementia. Clin. Biochem. 47(15), 51-53 (2014).

23. Rainesalo S, Keranen T, Saransaari P, Honkaniemi J. GABA and glutamate transporters are expressed in human platelets. Brain Res. Mol. Brain Res. 141(2), 161-165 (2005).

24. Vignini A, Sartini D, Morganti S et al. Platelet amyloid precursor protein isoform expression in Alzheimer's disease: evidence for peripheral marker. Int. J. Immunopathol. Pharmacol. 24(2), 529-534 (2011).

25. Manzine PR, Marcello E, Borroni B et al. ADAM10 gene expression in the blood cells of Alzheimer's disease patients and mild cognitive impairment subjects. Biomarkers 20(3), 196-201 (2015).

26. Prodan CI, Ross ED, Vincent AS, Dale GL. Coated platelets are higher in amnestic versus nonamnestic patients with mild cognitive impairment. Alzheimers Dis. Assoc. Disord. 21(3), 259-261 (2007).

27. Islam BR, Tabrez S. Management of Alzheimer's disease - an insight of the enzymatic and other novel potential targets. Int. J. Biol. Macromol. 97, 700-709 (2017).

28. Maqbool M, Mobashir M, Hoda N. Pivotal role of glycogen synthase kinase-3: a therapeutic target for Alzheimer's disease. Eur. J. Med. Chem. 107, 63-81 (2016).

29. Forlenza OV, Torres CA, Talib LL et al. Increased platelet GSK3B activity in patients with mild cognitive impairment and Alzheimer's disease. J. Psychiatr. Res. 45(2), 220-224 (2011).

30. Neumann K, Farías G, Slachevsky A, Perez P, Maccioni RB. Human platelets tau: a potential peripheral marker for Alzheimer's disease. J. Alzheimers Dis. 25(1), 103-109 (2011).

31. Fišar Z, Hroudová J, Hansíková $\mathrm{H}$ et al. Mitochondrial respiration in the platelets of patients with Alzheimer's disease. Curr. Alzheimer Res. 13(8), 930-941 (2016). 
32. Zhao S, Zhao J, Zhang T, Guo C. Increased apoptosis in the platelets of patients with Alzheimer's disease and amnestic mild cognitive impairment. Clin. Neurol. Neurosurg. 143, 46-50 (2016).

33. Wojsiat J, Laskowska-Kaszub K, Alquézar C et al. Familial Alzheimer's disease lymphocytes respond differently than sporadic cells to oxidative stress: upregulated p53-p21 signaling linked with presenilin 1 mutants. Mol. Neurobiol. 54(7), 5683-5698 (2016).

34. Veitinger M, Varga B, Guterres SB, Zellner M. Platelets, a reliable source for peripheral Alzheimer's disease biomarkers? Acta Neuropathol. Commun. 2, 65 (2014).

35. Vieira-de-Abreu A, Campbell RA, Weyrich AS, Zimmerman GA. Platelets: versatile effector cells in hemostasis, inflammation, and the immune continuum. Semin. Immunopathol. 34(1), 5-30 (2012).

36. Langer HF, Chavakis T. Platelets and neurovascular inflammation. Thromb. Haemost. 110(5), 888-893 (2013).

37. Canobbio I, Visconte C, Oliviero B et al. Increased platelet adhesion and thrombus formation in a mouse model of Alzheimer's disease. Cell Signal. 28(12), 1863-1871 (2016).

38. Casoli T, Di Stefano G, Balietti M, Solazzi M, Giorgetti B, Fattoretti P. Peripheral inflammatory biomarkers of Alzheimer's disease: the role of platelets. Biogerontology 11(5), 627-633 (2010).

39. Bermejo P, Martín-Aragón S, Benedí J et al. Differences of peripheral inflammatory markers between mild cognitive impairment and Alzheimer's disease. Immunol. Lett. 117(2), 198-202 (2008).

40. Gattaz WF, Talib LL, Schaeffer EL, Diniz BS, Forlenza OV. Low platelet iPLA 2 activity predicts conversion from mild cognitive impairment to Alzheimer's disease: a 4-year follow-up study. J. Neural. Transm. (Vienna) 121(2), 193-200 (2014).

41. Liu L, Zhang K, Tan L, Chen YH, Cao YP. Alterations in cholesterol and ganglioside GM1 content of lipid rafts in platelets from patients with Alzheimer disease. Alzheimer Dis. Assoc. Disord. 29(1), 63-69 (2015).

42. Tajeddinn W, Fereshtehnejad SM, Seed Ahmed M et al. Association of platelet serotonin levels in Alzheimer's disease with clinical and cerebrospinal fluid markers. J. Alzheimers Dis. 53(2), 621-630 (2016).

43. Wojda U, Kuznicki J. Alzheimer's disease modeling: ups, downs, and perspectives for human induced pluripotent stem cells. J. Alzheimers Dis. 34(3), 563-588 (2013).

44. Canobbio I, Abubaker AA, Visconte C, Torti M, Pula G. Role of amyloid peptides in vascular dysfunction and platelet dysregulation in Alzheimer's disease. Front. Cell. Neurosci. 9, 65 (2015).

45. Kucheryavykh LY, Dávila-Rodríguez J, Rivera-Aponte DE et al. Platelets are responsible for the accumulation of $\beta$-amyloid in blood clots inside and around blood vessels in mouse brain after thrombosis. Brain Res. Bull. 128, 98-105 (2017).

46. Wojsiat J, Prandelli C, Laskowska-Kaszub K, Martín-Requero A, Wojda U. Oxidative stress and aberrant cell cycle in Alzheimer's disease lymphocytes: diagnostic prospects. J. Alzheimer's Dis. 46(2), 329-350 (2015).

- An extensive review of potential biomarkers in lymphocytes.

47. Herrera-Rivero M, Soto-Cid A, Hernandez ME, Aranda-Abreu GE. Tau, APP, NCT and BACE1 in lymphocytes through cognitively normal aging and neuropathology. An. Acad. Bras. Cienc. 85(4), 1489-1496 (2013).

48. Arce-Varas N, Abate G, Prandelli C et al. Comparison of extracellular and intracellular blood compartments highlights redox alterations in Alzheimer's and mild cognitive impairment patients. Curr. Alzheimer Res. 14(1), 112-122 (2017).

49. Grimm A, Friedland K, Eckert A. Mitochondrial dysfunction: the missing link between aging and sporadic Alzheimer's disease. Biogerontology 17(2), 281-296 (2015).

50. Ponce DP, Salech F, SanMartin CD et al. Increased susceptibility to oxidative death of lymphocytes from Alzheimer patients correlates with dementia severity. Curr. Alzheimer Res. 11(9), 892-898 (2014).

51. Wang X, Wang W, Li L et al. Oxidative stress and mitochondrial dysfunction in Alzheimer's disease. Biochim. Biophys. Acta 1842(8), 1240-1247 (2014).

52. Bajic V, Spremo-Potparevic B, Zivkovic L, Isenovic ER, Arendt T. Cohesion and the aneuploid phenotype in Alzheimer's disease: a tale of genome instability. Neurosci. Biobehav. Rev. 55, 365-374 (2015).

53. Herrup K, Carrillo MC, Schenk D et al. Beyond amyloid: getting real about nonamyloid targets in Alzheimer's disease. Alzheimers Dement. 9(4), 452.e1-458.e1 (2013).

54. Zekanowski C, Wojda U. Aneuploidy, chromosomal missegregation, and cell cycle reentry in Alzheimer's disease. Acta Neurobiol. Exp. (Wars) 69(2), 232-253 (2009).

55. Kreis NN, Louwen F, Yuan J. Less understood issues: P21Cip1 in mitosis and its therapeutic potential. Oncogene 34(14), 1758-1767 (2015).

56. Esteras N, Alquezar C, Bermejo-Pareja F et al. Downregulation of extracellular signal-regulated kinase $1 / 2$ activity by calmodulin KII modulates p21Cip1 levels and survival of immortalized lymphocytes from Alzheimer's disease patients. Neurobiol. Aging 34(4), 1090-1100 (2013).

57. Buizza L, Prandelli C, Bonini SA et al. Conformational altered $\mathrm{p} 53$ affects neuronal function: relevance for the response to toxic insult and growth-associated protein 43 expression. Cell Death Dis. 4, e484 (2013). 
58. Bieging KT, Mello SS, Attardi LD. Unravelling mechanisms of p53-mediated tumour suppression. Nat. Rev. Cancer 14(5), 359-370 (2014).

59. Stieler J, Grimes R, Weber D et al. Multivariate analysis of differential lymphocyte cell cycle activity in Alzheimer's disease. Neurobiol. Aging 33(2), 234-241 (2012).

60. Alquezar C, Esteras N, de la Encarnacion A et al. PGRN haploinsufficiency increased Wnt5a signalingin peripheral cells from frontotemporal lobar degeneration-progranulin mutation carriers. Neurobiol. Aging 35(4), 886-898 (2014).

61. Bartolome F, Munoz U, Esteras $\mathrm{N}$ et al. Distinct regulation of cell cycle and survival in lymphocytes from patients with Alzheimer's disease and amyotrophic lateral sclerosis. Int. J. Clin. Exp. Pathol. 2(4), 390-398 (2009).

62. Heppner FL, Ransohoff RM, Becher B. Immune attack: the role of inflammation in Alzheimer disease. Nat. Rev. Neurosci. 16(6), 358-372 (2015).

63. Delaby C, Gabelle A, Blum D et al. Central nervous system and peripheral inflammatory processes in Alzheimer's disease: biomarker profiling approach. Front. Neurol. 6, 181 (2015).

64. van de Haar HJ, Burgmans S, Jansen JF et al. Blood-brain barrier leakage in patients with early Alzheimer disease. Radiology 281(2), 615 (2017).

65. Lucas HR, Rifkind JM. Considering the vascular hypothesis of Alzheimer's disease: effect of copper associated amyloid on red blood cells. Adv. Exp. Med. Biol. 765, 131-138 (2013).

66. van Oijen M, Hofman A, Soares HD, Koudstaal PJ, Breteler MM. Plasma Abeta (1-40) and Abeta(1-42) and the risk of dementia: a prospective case-cohort study. Lancet Neurol. 5(8), 655-660 (2006).

67. Kiko T, Nakagawa K, Satoh A et al. Amyloid $\beta$ levels in human red blood cells. PLoS ONE 7(11), e49620 (2012).

68. Nakagawa K, Kiko T, Kuriwada $S$ et al. Amyloid $\beta$ induces adhesion of erythrocytes to endothelial cells and affects endothelial viability and functionality. Biosci. Biotechnol. Biochem. 75(10), 2030-2033 (2011).

69. Lan J, Liu J, Zhao Z et al. The peripheral blood of A $\beta$ binding RBC as a biomarker for diagnosis of Alzheimer's disease. Age Aging 44(3), 458-464 (2015).

70. Lee NC, Yang SY, Chieh JJ et al. Blood beta-amyloid and tau in down syndrome: a comparison with Alzheimer's disease. Front. Aging Neurosci. 8, 316 (2017).

71. Iulita MF, Ower A, Barone C et al. An inflammatory and trophic disconnect biomarker profile revealed in down syndrome plasma: relation to cognitive decline and longitudinal evaluation. Alzheimers Dement. 12(11), 1132-1148 (2016).

72. Nakagawa K, Kiko T, Miyazawa T et al. Amyloid $\beta$-induced erythrocytic damage and its attenuation by carotenoids. FEBS Lett. 585(8) 1249-1254 (2011).

73. Skoumalova A, Ivica J, Santorova P et al. The lipid peroxidation products as possible markers of Alzheimer's disease in blood. Exp. Gerontol. 46(1), 38-42 (2011).

74. Chmatalova Z, Vyhnalek M, Laczo J, Hort J, Skoumalova A. Analysis of lipophilic fluorescent products in blood of Alzheimer's disease patients. J. Cell. Mol. Med. 20(7), 1367-1372 (2016).

75. Carelli-Alinovi C, Ficarra S, Russo AM et al. Involvement of acetylcholinesterase and protein kinase $\mathrm{C}$ in the protective effect of caffeine against $\beta$-amyloid-induced alterations in red blood cells. Biochimie 121, 52-59 (2016).

76. Kosenko EA, Aliev G, Kaminsky YG. Relationship between chronic disturbance of 2,3-diphosphoglycerate metabolism in erythrocytes and Alzheimer disease. CNS Neurol. Disord. Drug Targets 15(1), 113-123 (2016).

77. Schuchardt JP, Köbe T, Witte V et al. Genetic variants of the FADS gene clusterAre associated with erythrocyte membrane LC PUFA levels in patients with mild cognitive impairment. J. Nutr. Health Aging 20(6), 611-620 (2016).

78. Tan ZS, Harris WS, Beiser AS et al. Red blood cell omega-3 fatty acid levels and markers of accelerated brain aging. Neurology 78(9), 658-664 (2012). 
